\title{
Correlating Super-Resolution Microscopy and Transmission Electron Microscopy Reveals Multiparametric Heterogeneity in Nanoparticles
}

\author{
Citation for published version (APA): \\ Andrian, T., Delcanale, P., Pujals, S., \& Albertazzi, L. (2021). Correlating Super-Resolution Microscopy and \\ Transmission Electron Microscopy Reveals Multiparametric Heterogeneity in Nanoparticles. Nano Letters, \\ 21(12), 5360-5368. https://doi.org/10.1021/acs.nanolett.1c01666
}

DOI:

10.1021/acs.nanolett.1c01666

Document status and date:

Published: $14 / 06 / 2021$

\section{Document Version:}

Publisher's PDF, also known as Version of Record (includes final page, issue and volume numbers)

\section{Please check the document version of this publication:}

- A submitted manuscript is the version of the article upon submission and before peer-review. There can be important differences between the submitted version and the official published version of record. People interested in the research are advised to contact the author for the final version of the publication, or visit the $\mathrm{DOI}$ to the publisher's website.

- The final author version and the galley proof are versions of the publication after peer review.

- The final published version features the final layout of the paper including the volume, issue and page numbers.

Link to publication

\footnotetext{
General rights

- You may freely distribute the URL identifying the publication in the public portal. follow below link for the End User Agreement:

www.tue.nl/taverne

\section{Take down policy}

If you believe that this document breaches copyright please contact us at:

openaccess@tue.nl

providing details and we will investigate your claim.
}

Copyright and moral rights for the publications made accessible in the public portal are retained by the authors and/or other copyright owners and it is a condition of accessing publications that users recognise and abide by the legal requirements associated with these rights.

- Users may download and print one copy of any publication from the public portal for the purpose of private study or research.

- You may not further distribute the material or use it for any profit-making activity or commercial gain

If the publication is distributed under the terms of Article $25 \mathrm{fa}$ of the Dutch Copyright Act, indicated by the "Taverne" license above, please 


\title{
Correlating Super-Resolution Microscopy and Transmission Electron Microscopy Reveals Multiparametric Heterogeneity in Nanoparticles
}

\author{
Teodora Andrian, Pietro Delcanale, Silvia Pujals,* and Lorenzo Albertazzi* \\ Cite This: Nano Lett. 2021, 21, 5360-5368 \\ Read Online
}

ABSTRACT: The functionalization of nanoparticles with functional moieties is a key strategy to achieve cell targeting in nanomedicine. The interplay between size and ligand number is crucial for the formulation performance and needs to be properly characterized to understand nanoparticle structure-activity relations. However, there is a lack of methods able to measure both size and ligand number at the same time and at the single particle level. Here, we address this issue by introducing a correlative light and electron microscopy (CLEM) method combining super-resolution

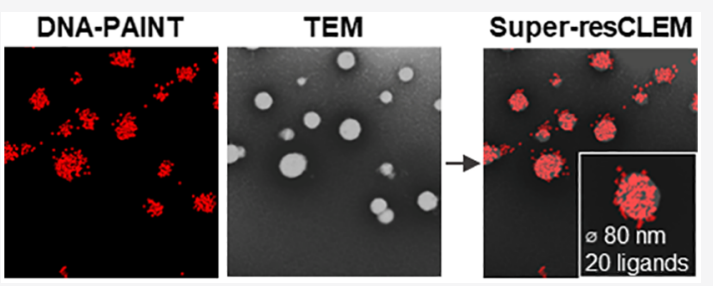
microscopy (SRM) and transmission electron microscopy (TEM) imaging. We apply our super-resCLEM method to characterize the relationship between size and ligand number and density in PLGA-PEG nanoparticles. We highlight how heterogeneity found in size can impact ligand distribution and how a significant part of the nanoparticle population goes completely undetected in the single-technique analysis. Super-resCLEM holds great promise for the multiparametric analysis of other parameters and nanomaterials.

KEYWORDS: super-resolution microscopy (SRM), electron microscopy (EM), correlative light and electron microscopy (CLEM), nanomedicine, nanoparticles, heterogeneity

$\mathrm{T}$ he field of nanomedicine is rapidly expanding in light of its expected impact on health care. ${ }^{1-3}$ Nanoparticles (NPs) conjugated with functional ligands have been developed for various applications, including imaging and diagnosis, ${ }^{4,5}$ and targeted drug delivery. ${ }^{6,7}$ Yet, despite many optimization efforts only a small fraction of the injected dose has shown to reach the target site, ${ }^{8}$ exposing the gap in our understanding of how the properties of ligand functionalized NPs can affect their biological responses. Common methods to characterize ligand functionalized NPs rely on averaged results, which do not provide an accurate picture of the material at a single-particle level, and generally underestimate the magnitude of heterogeneity in ligand number and distribution.

It is particularly important to study the heterogeneity in size and functional ligand distribution as they are the main determinants of the formulation's in vivo fate. First, NP size is a major determinant of cellular uptake, ${ }^{10,11}$ blood circulation half-life, ${ }^{12,13}$ biodistribution, ${ }^{14,15}$ tumor permeability ${ }^{16}$ and immune response. ${ }^{17}$ Second, the functionalization of NP surface with targeting ligands is the most used strategy to achieve tissue and cell-selective delivery of drug carriers through the recognition of biomarkers on the cell surface. In this context, ligand number, affinity, and distribution govern the NP biodistribution, cell selectivity, and internalization and as a consequence its therapeutic efficiency. ${ }^{18,19}$

Size is generally characterized by dynamic light scattering (DLS), while electron microscopy (EM) and atomic force microscopy (AFM) are used to reinforce the results as they can provide direct characterization of the size distribution and morphology of nanomaterials at the single particle level. ${ }^{20-22}$ On the contrary, quantification of ligand numbers and ligand distribution proves to be more challenging and it is often carried out with indirect assays based on averaged values which mask the heterogeneity in a nanoparticle formulation. ${ }^{9,23,24}$ Moreover, analysis at a single particle level with high throughput is still suffering from a lack of accurate and standardized techniques. ${ }^{23}$

Recently, super-resolution microscopy (SRM) techniques based on single-molecule localization (SMLM) have been used for the analysis and quantification of synthetic nanomaterials in vitro and within cells, ${ }^{25-29}$ as well of functional ligands, ${ }^{25,30,31}$ thanks to their superior resolution $(10-20 \mathrm{~nm})$, molecular specificity, and sensitivity. ${ }^{32}$ DNA Points Accumulation for Imaging in Nanoscale Topography (DNA-PAINT), a type of SMLM technique, ${ }^{33}$ has been applied to map the functional sites on the surface of polystyrene NPs and to explore the spatial distribution and surface heterogeneity of the active sites on their surface. ${ }^{25}$ DNA-PAINT can be used to quantify single molecules (i.e., molecular counting) and achieves high multiplexing, low photobleaching, and is accurate for a wide

Received: April 27, 2021

Revised: June 3, 2021

Published: June 14, 2021 


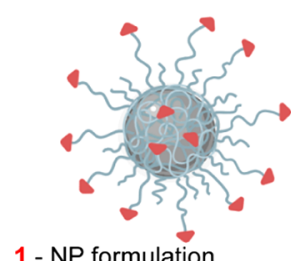

1 - NP formulation

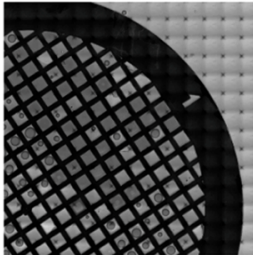

4 - Reference acquisition

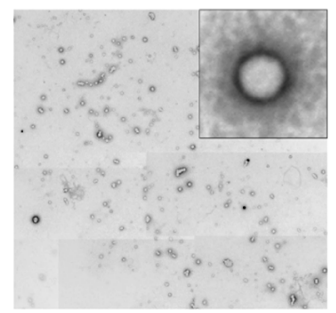

7 - TEM imaging, stitching and size quantification

^ PEG $1 k$

$\sim$ PEG5k-Maleimide

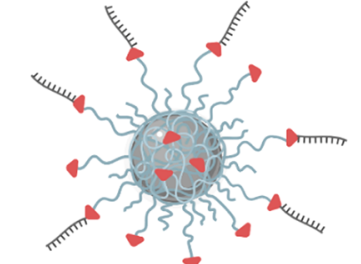

2 - Tagging ligands with DNA

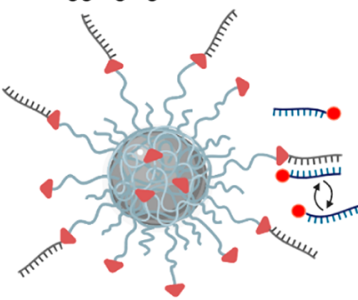

5 - DNA-PAINT imaging

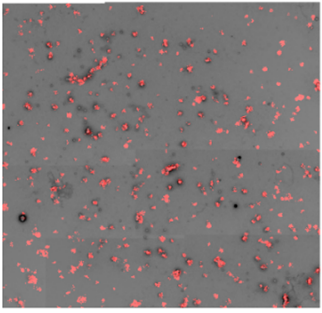

8 - Image correlation

TIIIIIT Docking strand (thiol-DNA oligonucleotide, ligand)

ITIIIT- Imager strand (complementary strand + Atto647N Dye)

Figure 1. Overview of the super-resCLEM method. Formulation of PLGA-PEG NPs via nanoprecipitation (1). Conjugation of NP maleimide groups to thiol-DNA 9-mer oligonucleotides (docking strands) acting as functional ligands (2). NPs are attached to a copper carbon-coated 200 mesh TEM grid, which is assembled into a chamber, followed by flow of complementary imager strand buffer solution (3). A reference image of the region of interest (ROI) is taken using a stitching function (4). Then the DNA-PAINT image is acquired, through transient binding and unbinding of the complementary imager strands attached to ATTO-647- $\mathrm{N}$ fluorophore (5). The txt. file consisting of the $x, y, t$ localizations coordinates is extracted and analyzed into number of localizations per NP. The exact number of available ligands is quantified using qPAINT analysis (6). For TEM imaging, the NP-coated TEM grids are negatively stained with $2 \%$ uranyl acetate; using the low-magnification function of a TEM microscope the ROI is found on the grid and imaged sequentially at $20000 \times$ magnification. The sequential images are manually stitched, and NP size can be quantified (7). DNA-PAINT and TEM images are correlated manually (8). Size and ligand number are quantified and correlated at a single-particle level (9). Schematic NP and arrow in (9) were created with BioRender.com.

range of functionalization densities. ${ }^{33,34}$ Quantitative PAINT (qPAINT), a technique originally used to quantify docking strands in DNA origami, ${ }^{35}$ can quantify the exact number of functional ligands on the surface of NPs, ${ }^{25,31,36}$ highlighting the applicability of SMLM in nanomedicine research.

Still, despite the advances in SRM that allow us to characterize NPs at a single particle level, we are only able to study the population of NPs that are labeled and thus lose information on NP size and morphology. Consequently, the relationship between various physiochemical properties (i.e., multiparametric) such as size and ligand number and distribution remains unclear. Although it is good practice to characterize samples with multiple techniques, a correlation between individual physiochemical parameters and biological performance cannot be made, as the effects of different parameters are entangled. ${ }^{37,38}$

Correlative light and electron microscopy (CLEM) are a powerful and well-established group of multimodal imaging systems that combine the benefits of both microscopies through detailed images of the same region. ${ }^{39}$ CLEM has proven its potential in structural biology ${ }^{40-45}$ and recently to track and quantify NPs intracellularly, ${ }^{46,47}$ but to the best of our knowledge it has not yet been explored for the structural characterization of nanomaterials. To address this issue, we have developed a correlative super-resolution microscopy (SRM) and transmission electron microscopy (TEM) (super-resCLEM) method. It combines the ability of SRM to quantify the number of surface ligands with the potential of TEM to characterize the size and morphology with nanometric precision and at a single particle level.

Here, we propose a super-resCLEM workflow for the characterization of functionalized polymeric poly(lactide-coglycolide)-poly(ethylene glycol) PLGA-PEG NPs. Polymeric NPs have been applied in targeted drug delivery systems due to their biocompatibility, biodegradability, and general ease in surface customization. ${ }^{48-50}$ A common strategy for surface grafting of NPs with targeting ligands is by surface modification with the spacer PEG, ${ }^{, 1}$ which also offers stealth behavior. $^{52,53}$ In this work, we functionalized PLGA-PEGmaleimide chains to our oligonucleotide ligand via a maleimide-thiol conjugation as this approach provides high reactivity and good final stability under most conditions. ${ }^{54-56}$

We first describe our super-resCLEM method and show its applicability in investigating the relationship between ligand number, ligand distribution, and ligand density versus size at a single-particle level and with nanometric resolution. We 

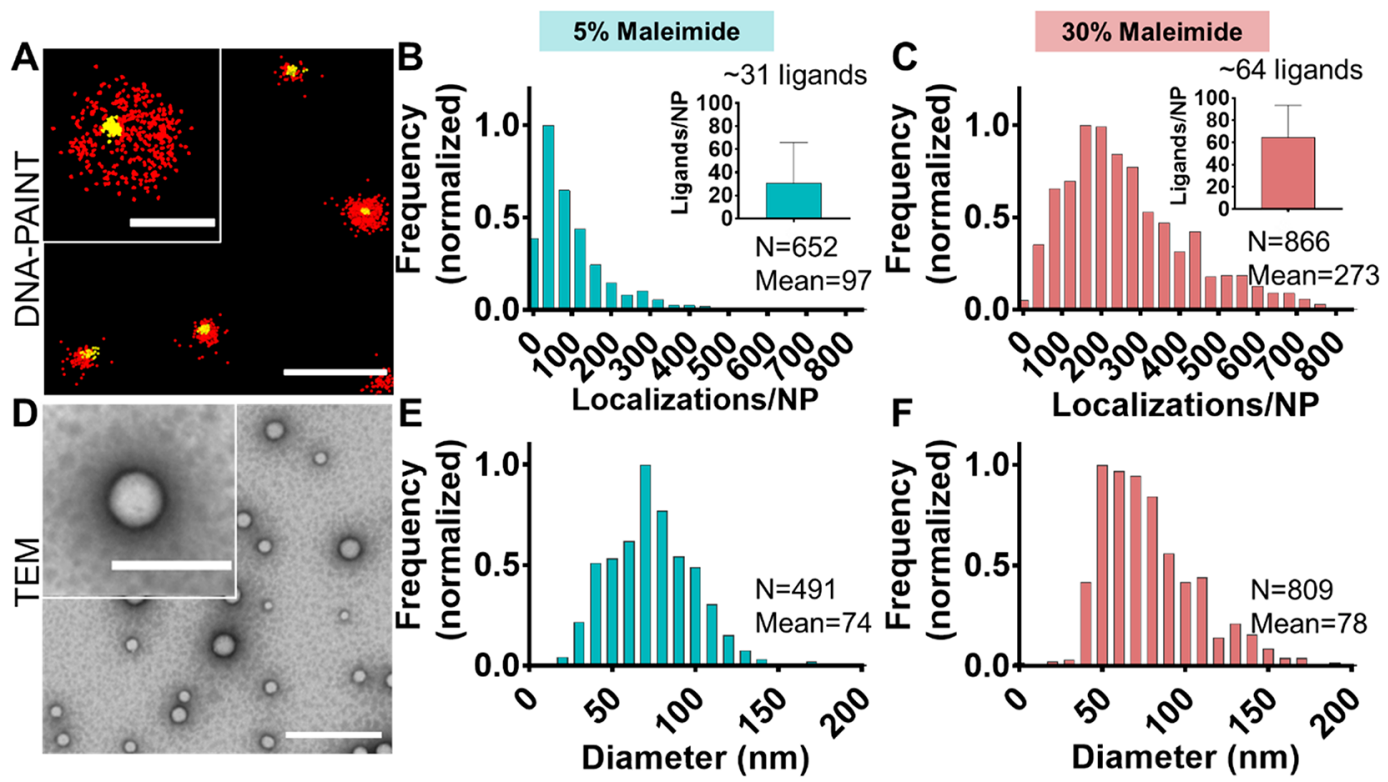

Figure 2. Characterization of localization distribution and ligand number and diameters in PLGA-PEG nanoparticles using DNA-PAINT and TEM. (A) DNA-PAINT images of PLGA-PEG 30\% maleimide NPs conjugated to thiol-docking strands in a large field (scale bar $1000 \mathrm{~nm}$ ) and a small field (upper left, scale bar $100 \mathrm{~nm}$ ). DNA-PAINT localizations are shown in red and DiI signal used for drift correction and as a reference in yellow. Normalized frequency histograms of DNA-PAINT localizations per NP for PLGA-PEG 5\% (B) and 30\% maleimide (C) formulations, including the number of NPs analyzed $(N)$ and the mean number of localizations per NP (Mean), as well as a bar graph depicting the number of ligands per NP quantified with qPAINT, and the average number of ligands per NP. Bin widths $=40$. Experimental details of DNA-PAINT imaging on glass can be found in Supporting Information. Experimental information on qPAINT on glass can be found in Figure S5. (D) Transmission electron microscopy (TEM) images of PLGA-PEG (30\% maleimide) NPs conjugated to thiol-docking strands in a large field (scale bar $1000 \mathrm{~nm}$ ) and a small field (upper left, scale bar $200 \mathrm{~nm}$ ). Normalized frequency histograms of NP diameter (nm) for PLGA-PEG 5\% (E) and 30\% maleimide (F) formulations, including the number of NPs analyzed $(N)$ and mean diameter in $\mathrm{nm}(\mathrm{Mean})$. Bin width $=10$.

surprisingly discover the presence of a large population of NPs with no ligands on their surface, as well as "invisible nanoparticles" that go undetected by DNA-PAINT imaging alone. Finally, we quantify the amount of accessible surface ligands per particle using our multiparametric correlative method and demonstrate its advantage over a one-method-ata-time approach. The applicability of our correlative method spans to a plethora of other different nanomaterials with the only requirement being the attachment of docking strands to the ligands of interest, although other DNA-free PAINT approaches could also be used. ${ }^{57,58}$ Therefore, our approach holds great promise for the multiparametric analysis of various other parameters and nanomaterials.

\section{RESULTS AND DISCUSSION}

Introducing Super-resCLEM Methodology. Our superresCLEM method is outlined in Figure 1, Materials and Methods, and Figure S1. Polymeric NPs were formulated manually via the nanoprecipitation method $^{59}$ using combinations of PLGA-PEG, PLGA, and PLGA-PEG-maleimide polymers (1). Then, NPs were conjugated to functional ligands through a thiol-maleimide reaction. ${ }^{58,60}$ The ligand consists of a thiol group conjugated to a short (nine bases) oligonucleotide strand (i.e., docking strand) (2). The NPs were adsorbed onto a carbon-coated copper TEM grid and prepared into a glass imaging chamber. Then, the complementary oligonucleotide strand labeled with Atto-647N (i.e., imager strand) was flown into the chamber (3). To relocate the region of interest (ROI) later in TEM, we collected large field-of-view bright field (BF) images of the grid to distinguish its orientation (4). In DNA-PAINT imaging, DNA hybridization drives the transient binding of the imager strands to the docking strands on the surface of the NPs, leading to fluorescence signal and localization of single molecules over thousands of frames ${ }^{33}(5)$. After image acquisition, space-time coordinates of individual molecules are analyzed to precisely quantify the number of available surface ligands per NP through the quantitative PAINT method (qPAINT) $25,31,35$ (6). Following negative staining, the grid was transferred to TEM, for size analysis and morphological inspection. Using the reference images, the ROI was established and sequentially imaged, then the single images stitched to create a "TEM canvas" of the ROI (7). Then, the SRM image was scaled and rotated to match the size and orientation of the TEM stitched image, then manually correlated to obtain a TEM canvas with overlapping clusters of localizations per single particle (8). The number of surface ligands and size of each NP was correlated at a single-particle level (9).

Characterization of NPs at a Single-Particle Level. We first tackle the characterization of PLGA-PEG NPs with DNA-PAINT and TEM separately. We formulated NPs with $5 \%$ and $30 \%$ maleimide content and conjugated them to an excess of ligand and used DNA-PAINT and qPAINT to quantify and analyze the ligand number and distribution. For further characterization, see Table S1, Figure S2, and Figure S3. To demonstrate that the DNA-hybridization is specific between the docking strand and imager strand, we carried out control experiments whereby the formulations were imaged under the same conditions using a noncomplementary imager strand (Figure S4).

Figure 2A shows a reconstructed DNA-PAINT image where the functional groups are imaged (red localizations). The yellow signal represents the encapsulated DiI dye, used as a reference in DNA-PAINT. NPs without the corresponding DiI 
signal are disregarded as unspecific signal. Intraparticle heterogeneity in surface ligand distribution is clear as every NP shows a distinct number of events. In Figure 2B,C, a quantification of the number of localizations/NP and the relative quantified number of ligands per NP by qPAINT is presented. The results for PLGA-PEG 5\% (Figure 2B) and $30 \%$ maleimide (Figure 2C) formulations reflect the expected increase in relation between maleimide content and number of localizations and/or ligands. Both formulations show nonsymmetrical localization distributions, with a broader distribution (i.e., more heterogeneous) at the higher maleimide content. By calculating the coefficient of variation (CV) of number of localizations per NP, we found that NPs formulated in the same way and in the same batch display a number of localizations that spans by $60-90 \%$ from the mean value, highlighting marked ligand heterogeneity in these formulations (Figure S6). Notably, DNA-PAINT is a fully random process, as the DNA strand molecules in solution have equal probability to attach to a complementary strand on an NP. ${ }^{30}$ The conjugation process of ligands to maleimide groups on NP surface is also expected to be stochastic, unlike the distributions observed here. A possible reason is that the stochastic process of ligand conjugation is entangled with other parameters, such as size, resulting in non-Poissonian distributions.

We therefore used TEM to study NP size heterogeneity. A typical TEM image depicting PLGA-PEG NPs is seen in Figure 2D. Figure $2 \mathrm{E}$ and Figure $2 \mathrm{~F}$ show the distributions in diameter at a single-particle level for the PLGA-PEG 5\% and $30 \%$ formulations, respectively. Although a nearly symmetrical distribution is seen for particles formulated with 5\% maleimide content, at $30 \%$ we observe a more heterogeneous distribution, similar to that observed in localizations per NP with DNAPAINT.

Observing heterogeneity in both size and functional ligands, we next correlated DNA-PAINT with TEM images to identify a possible relationship between the two parameters at a singleparticle level.

Multiparametric Characterization of NPs Using Super-resCLEM. In Figure 3, we introduce a representative

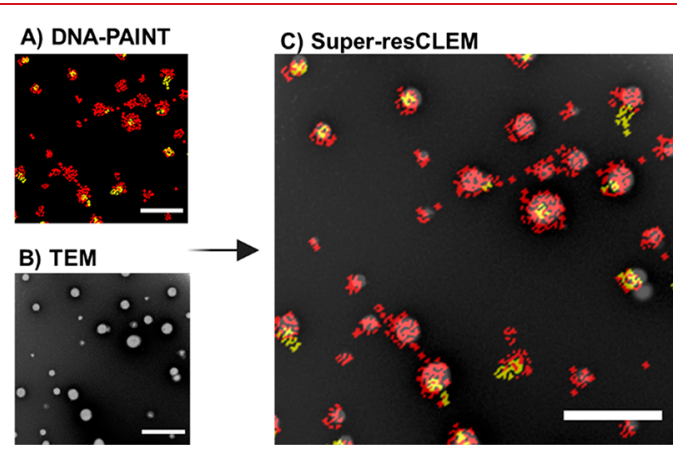

Figure 3. Correlative DNA-PAINT and TEM (super-resCLEM) image of PLGA-PEG nanoparticles. (A) DNA-PAINT image where red localizations are representative of ligand number and yellow localizations of encapsulated DiI dye used as a reference marker and (B) TEM image, both corresponding to the same PLGA-PEG $30 \%$ maleimide NPs. (C) Overlaid super-resCLEM image. NPs without the reference DiI signal ("invisible particles") would be discarded in DNA-PAINT imaging alone. All scale bars $=500 \mathrm{~nm}$. For details of image acquisition and data analysis see Materials and Methods. Arrow was created with BioRender.com. correlative image obtained using our proposed super-resCLEM method on ligand conjugated PLGA-PEG NPs. We first obtained a DNA-PAINT image (Figure 3A) prior to sample preparation required for TEM, to preserve the surface docking strands intact for the hybridization with the complementary imaging strands. Particles are visible as red clusters of localizations, representative of the number of surface ligands, with an appreciable heterogeneity among them. Then, a TEM image was acquired on the same field of view (Figure 3B), clearly highlighting NPs of different sizes. The merging of these two images results in the final super-resCLEM image (Figure 3C), which allows us to make two important qualitative observations: a marked heterogeneity in both number of localizations and size per NP, and the presence of particles without the reference DiI signal, that would otherwise be invisible to DNA-PAINT imaging alone (i.e., "invisible particles").

Using the correlative images for both $5 \%$ and $30 \%$ formulations (Figure 4A,B, respectively), we studied the relationship between the number of ligands per NP versus TEM diameter at a single particle level for both formulations (Figure 4C,F, respectively). By observing these scatterplots, where every NP is one cross, we see that the number of surface ligands per NP increases exponentially with increasing NP size but also that both formulations display heterogeneity in the trend as shown by the broad data clouds. To better understand the trend, particles were binned according to their diameter and the average ligand number was obtained for particles within each bin (Figure 4C,F, black circles). The obtained averaged data are well fitted with a power model (Figure 4C,F, gray line). The results demonstrate that the number of ligands per NP increases roughly as a power of 2 (1.8 for 5\% and 2.5 for $30 \%$ maleimide formulations) with increasing diameter. This suggests that the number of ligands is directly proportional to the area of a particle, approximated as a sphere. Although the averaged bins clearly follow the power law, the single particle data (i.e., the crosses) show a much broader relationship, notably, with more heterogeneity observed for NPs with $30 \%$ maleimide content and greater than $120 \mathrm{~nm}$ in diameter. For more statistical information see Figure S7. Next, we plotted a scatter graph of ligand density per NP versus diameter for both formulations. At 5\% maleimide (Figure 4D), NPs with diameters between 50 and $120 \mathrm{~nm}$ show the expected trend between these parameters, that is, the number of ligands per $\mu \mathrm{m}^{2}$ does not generally change with diameter. A similar trend is observed at the $30 \%$ maleimide (Figure 4G) albeit with a much broader heterogeneity, particularly for diameters $>120 \mathrm{~nm}$. In both formulations, we also observe a cloud of NPs with 0 ligand density for nearly all NP sizes.

To better understand these results, we analyzed the distributions of ligands per NP for smaller (0-99 nm) and larger (100-200 nm) NP populations for both formulations. We noted that the ligand distribution is more heterogeneous at $30 \%$ maleimide (Figure $4 \mathrm{H}$ ) than at 5\% maleimide (Figure 4E) content, particularly in the larger size population, similar to the results observed in the relationship between ligand number and ligand density versus diameter. These findings, as well as other previously published studies ${ }^{61,62}$ may suggest that heterogeneity found in NP size can affect the surface composition and, as described here, disrupt the expected trends in ligand number and in ligand density. Consequently, the presence of NP populations with distinct physiochemical 


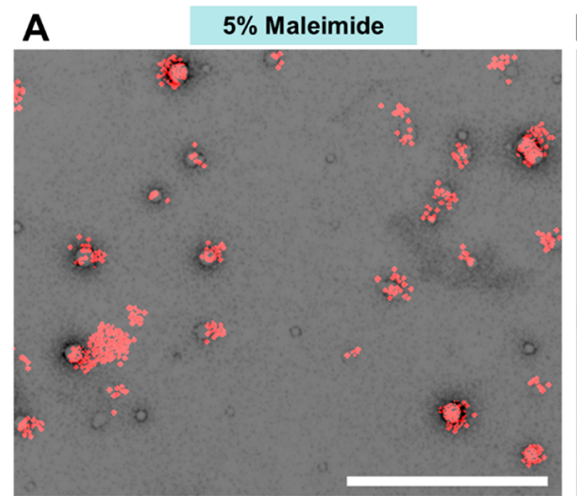

B $\quad 30 \%$ Maleimide

C

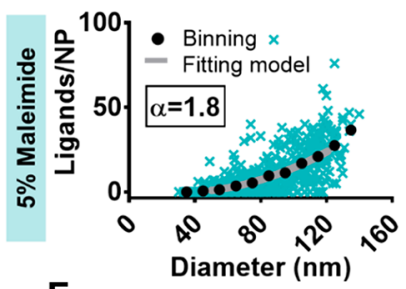

$\mathbf{F}$
D

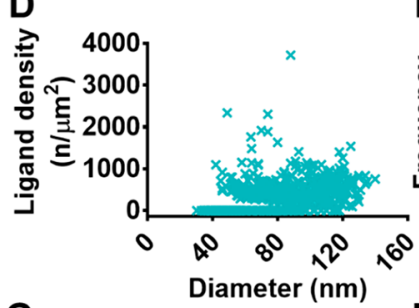

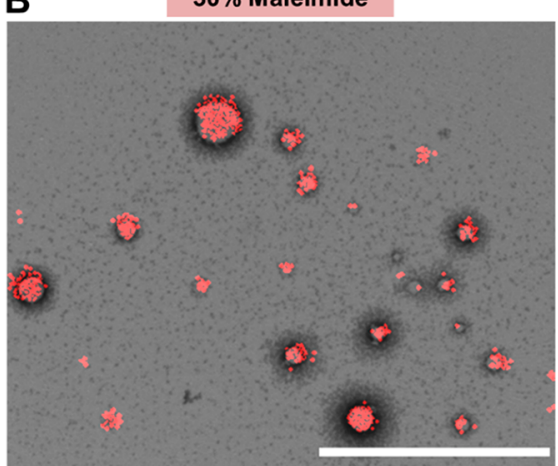

E
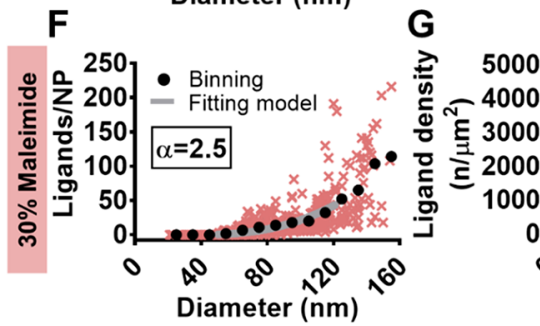

Diameter $(\mathrm{nm}) \mathrm{H}$
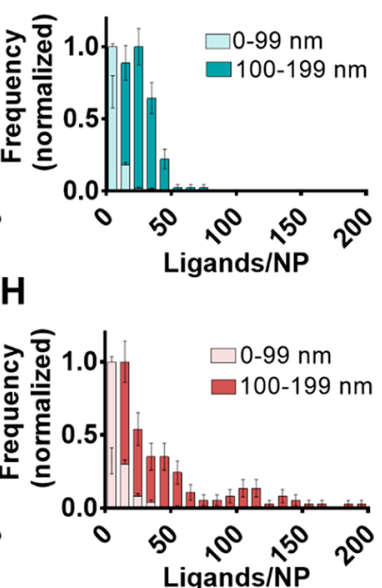

Figure 4. Multiparametric characterization of PLGA-PEG nanoparticles using super-resCLEM. Super-resCLEM image of (A) PLGA-PEG 5\% (A) and 30\% maleimide (B) nanoparticle formulations (scale bars $=1000 \mathrm{~nm}$ ). The relationship between the number of ligands per NP as quantified by qPAINT, and the corresponding diameter as measured by TEM of PLGA-PEG 5\% (C) and 30\% maleimide (F) formulations. Black dots show the same data binned on TEM diameter (bin size $10 \mathrm{~nm}$ ) where the average number of ligands is shown for each bin. Gray lines show the results of the fitting of binned data with a power-law model. The relationship between ligand density (number of ligands per NP surface area in $\mu \mathrm{m}^{2}$ ) and the corresponding diameter by TEM for PLGA-PEG 5\% (D) and 30\% maleimide (G) NP formulations. Distributions of ligand number per NP based on diameter ranges by TEM of 0-99 nm and 100-199 nm for PLGA-PEG 5\% (E) and 30\% maleimide (H) formulations. Note: the DiI signal is not present in the CLEM images in A and B as it was not used as a reference in the analysis. In this case, TEM is used to confirm true NPs in DNA-PAINT. Details of image acquisition, data analysis, and surface area calculation can be found in Materials and Methods.

properties in the same batch can lead to different outcomes in therapeutic efficacy. ${ }^{23}$

Super-resCLEM endows us with the possibility to study the whole NP population at a single particle level, including those NPs without any reference signal that would otherwise be invisible if analyzed solely by SRM as depicted in Figure 3. To better understand the various subpopulations within our formulations, we subdivided the whole NP population according to the quantified number of ligands on their surface and then calculated the percentage of each subpopulation with respect to the total amount of NPs. We found a remarkably large percentage of NPs without any functional ligands: $42 \%$ and $28 \%$ at $5 \%$ (Figure $5 \mathrm{~A}$ ) and $30 \%$ (Figure 5B) maleimide contents, respectively. Similar results were observed also on dendrimers, whereby over $45 \%$ of the entire material showed no surface ligands and very heterogeneous populations, ${ }^{9,63}$ which opens up the door to a multitude of questions regarding the performance of these nanoparticle subpopulations that are in fact nonfunctional, which could also lead to toxicity and undesirable biological immune responses. ${ }^{23}$

We then compared our results for the number of ligands per NP with the average theoretical calculations that are normally used in literature (Table 1). First, we calculated the
A
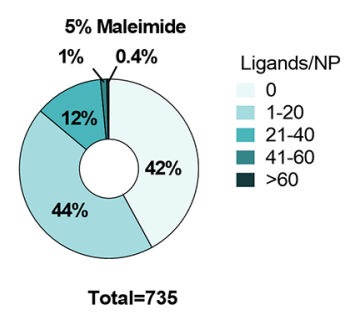

B

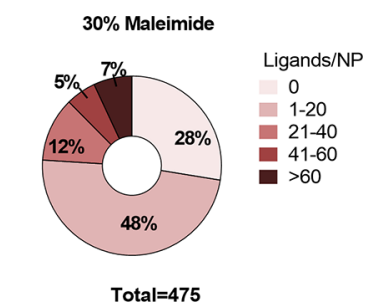

Figure 5. Pie charts depicting NP populations (\%) with 0, 1-20, 21$40,41-60$ or $>60$ ligands/NP in PLGA-PEG 5\% (A) and 30\% maleimide (B) NP formulations, as quantified and analyzed by superresCLEM. The total number of NPs analyzed per formulation is shown below each pie chart.

conjugation efficiency (CE \%) of our NP formulations to the functional ligands (Table 1) and as a comparison to the smaller molecule cysteine through a cysteine assay (Table S2) and found the $\mathrm{CE}$ (\%) values to be between 23 and $70 \%$, suggesting that the number of accessible ligands is overestimated. Theoretical calculations assume that all the hydrophilic PEG-maleimide chains will migrate and be exposed to the NP surface, while the hydrophobic PLGA will form the core. However, due to the miscibility of PEG and PLGA ${ }^{64,65}$ 
Table 1. Calculation of Ligand Availability (\%) Values Using DNA-PAINT Alone and Super-resCLEM ${ }^{a}$

\begin{tabular}{|c|c|c|c|c|c|c|}
\hline formulation & $\begin{array}{c}\text { diameter TEM } \\
(\mathrm{nm})\end{array}$ & $\begin{array}{l}\text { theoretical maleimide } \\
\text { molecules/NP }\end{array}$ & $\begin{array}{l}\mathrm{CE} \\
(\%)\end{array}$ & $\begin{array}{l}\text { theoretical ligand } \\
\text { number/NP }\end{array}$ & $\begin{array}{l}\text { accessible ligands } \\
\text { (qPAINT) }\end{array}$ & $\begin{array}{c}\text { ligand availability } \\
(\%)\end{array}$ \\
\hline PLGA-PEG 5\% Mal. & $74 \pm 26$ & 321 & 23 & 49 & 26 & 47 \\
\hline PLGA-PEG 30\% Mal. & $78 \pm 29$ & 1630 & 70 & 1141 & 54 & 5 \\
\hline PLGA-PEG 5\% Mal. & $77 \pm 25$ & 357 & 23 & 83 & 8 & 10 \\
\hline PLGA-PEG 30\% Mal. & $82 \pm 31$ & 2746 & 70 & 1922 & 19 & 1 \\
\hline
\end{tabular}

${ }^{a}$ Average TEM diameter, theoretical maleimide molecules per NP, conjugation efficiency (CE \%), theoretical ligand number per NP, accessible ligands per NP as quantified by qPAINT, and resulting average ligand availability (\%) for PLGA-PEG 5\% and 30\% maleimide formulations. These results were obtained either by a one-method-at-a-time approach using qPAINT and TEM separately (top two rows) or by a correlative method using super-resCLEM (bottom two rows). For calculations, see Materials and Methods.

the NP core in fact also includes PEG-maleimide chains. It has been estimated that only about $50-60 \%$ of the maleimide groups added are available for conjugation on the NP surface, ${ }^{56}$ values in close accordance with our results. We then calculated the ligand availability (\%) (i.e., the percentage of surface ligands per NP accessible to imager strands) for both formulations with the results obtained by a one-method-at-atime approach using TEM and qPAINT data separately (Table 1 , top two rows) and compared these with the results calculated by super-resCLEM (Table 1 , bottom two rows). The ligand availability (\%) is generally lower than the CE (\%), meaning that not all conjugated surface ligands are accessible to the imager strands, which could be due to various reasons. First, there is still a risk of ligand embedding in the PEG brush after conjugation, ${ }^{66,67}$ and second the functional ligands could be poorly orientated to the imager strands. Lastly, since our functional ligands and imager strands are DNA based and their negative charges could lead to an electrostatic repulsion between the strands, hindering a close packed arrangement, especially at a greater maleimide content, as seen in our results.

We observed a general 5-fold overestimation in ligand availability (\%) with a one-method-at-a-time approach compared to our super-resCLEM method. Using the latter approach, we are not limited to only imaging the population of NPs with a reference signal, but are indeed able to analyze the whole population, including the "invisible particles" as generally all particles are visible in TEM. As depicted in Table 1, a single parametric approach would also overemphasize the therapeutic performance of the formulation and prevent the determination of a sufficiently effective NP dose.

\section{CONCLUSIONS}

In the present work, we introduce an efficient method based on super-resCLEM imaging to study the relationship between size and ligand number and density at a single particle level with nanometric resolution. First, we demonstrate that the heterogeneity found in NP parameters may be a result of the collective impact between different physiochemical properties such as size and surface ligand number. Second, we found a remarkable percent of NPs without any surface ligands, which in a therapeutic formulation would be ineffective and could even lead to toxicity and undesirable biological immune responses. Third, we show that the characterization of nanomaterials using a one-method-at-a-time approach limits the information obtained as compared to a multiparametric technique. For example, omitting subpopulations of NPs with no reference signal and importantly no surface ligands. The latter leads to an overestimation of the number of ligands and ligand availability (\%) as observed by DNA-PAINT alone, which in hindsight would prevent the determination of an adequately efficient therapeutic dose. The study of other nanomaterials using super-resCLEM, particularly with different morphologies, would shine light on the relationship between size and morphology and surface functionalization. Although the multiparametric characterization of the tens of different physiochemical properties relevant to nanomaterial performance is still out of our reach, we hope that this work will pave the way to a more robust characterization using correlative imaging techniques.

\section{ASSOCIATED CONTENT}

\section{Supporting Information}

The Supporting Information is available free of charge at https://pubs.acs.org/doi/10.1021/acs.nanolett.1c01666.

Materials and methods; NP formulation and ligand conjugation; calculations of ligand density per NP; full CLEM protocol; calculation of conjugation efficiency and theoretical maleimide groups per NP, calculations of theoretical ligands per NP and ligand availability; DNAPAINT measurements on glass; cysteine assay protocol; complementary schematic information on CLEM protocol; full NP characterization using DLS, zeta potential and DNA-PAINT; DNA-PAINT negative controls; extra statistical analysis; cysteine assay results (PDF)

\section{AUTHOR INFORMATION}

\section{Corresponding Authors}

Lorenzo Albertazzi - Institute for Bioengineering of Catalonia (IBEC), The Barcelona Institute of Science and Technology, 08028 Barcelona, Spain; Department of Biomedical Engineering, Institute for Complex Molecular Systems (ICMS), Eindhoven University of Technology, 5612AZ Eindhoven, The Netherlands; orcid.org/0000-00026837-0812; Email: 1.albertazzi@tue.nl, lalbertazzi@ ibecbarcelona.eu

Silvia Pujals - Institute for Bioengineering of Catalonia (IBEC), The Barcelona Institute of Science and Technology, 08028 Barcelona, Spain; Department of Electronics and Biomedical Engineering, Faculty of Physics, Universitat de Barcelona, 08028 Barcelona, Spain; Email: spujals@ ibecbarcelona.eu

\section{Authors}

Teodora Andrian - Institute for Bioengineering of Catalonia (IBEC), The Barcelona Institute of Science and Technology, 08028 Barcelona, Spain

Pietro Delcanale - Dipartimento di Scienze Matematiche, Fisiche e Informatiche, Università di Parma, 43124 Parma, Italy 
Complete contact information is available at:

https://pubs.acs.org/10.1021/acs.nanolett.1c01666

\section{Author Contributions}

The manuscript was written through contributions of all authors.

\section{Notes}

The authors declare no competing financial interest.

\section{ACKNOWLEDGMENTS}

This work was supported by a Marie Skłodowska-Curie Horizon 2020 (713673), "la Caixa” Foundation (ID 100010434, code LCF/BQ/DI18/11660039) fellowship. S.P. and L.A acknowledge the financial support by the Spanish Ministry of Science and Innovation (PID2019-109450RB-I00/ AEI/10.13039/501100011033), European Research Council/ Horizon 2020 (ERC-StG-757397), "la Caixa" Foundation (ID 100010434), and by the Generalitat de Catalunya (through the CERCA program and 2017 SGR 01536). L.A. thanks the support of NWO through the VIDI Grant 192.028. The authors thank Lidia Delgado Valderrama and Maria Yolanda Muela Castro from the Electron Cryomicroscopy Unit from CCiTUB.

\section{REFERENCES}

(1) Jain, K. Future of nanomedicine: impact on healthcare \& society. Nanomedicine 2015, 10 (21), 3199-3202.

(2) Li, Z.; Tan, S.; Li, S.; Shen, Q.; Wang, K. Cancer drug delivery in the nano era: An overview and perspectives. Oncol. Rep. 2017, 38 (2), 611-624.

(3) Ventola, C. L. Progress in Nanomedicine: Approved and Investigational Nanodrugs 2017, 42 (12), 742-755.

(4) Martinelli, C.; Pucci, C.; Ciofani, G. Nanostructured carriers as innovative tools for cancer diagnosis and therapy. APL Bioengineering 2019, 3 (1), 011502.

(5) Loo, C.; Lowery, A.; Halas, N.; West, J.; Drezek, R. Immunotargeted Nanoshells for Integrated Cancer Imaging and Therapy. Nano Lett. 2005, 5 (4), 709-11.

(6) Pearce, A. K.; O’Reilly, R. K. Insights into Active Targeting of Nanoparticles in Drug Delivery: Advances in Clinical Studies and Design Considerations for Cancer Nanomedicine. Bioconjugate Chem. 2019, 30 (9), 2300-2311.

(7) Karra, N.; Benita, S. The Ligand Nanoparticle Conjugation Approach for Targeted Cancer Therapy. Curr. Drug Metab. 2012, 13 (1), 22-41.

(8) Wilhelm, S.; Tavares, A. J.; Dai, Q.; Ohta, S.; Audet, J.; Dvorak, H. F.; Chan, W. C. W.; et al. Analysis of nanoparticle delivery to tumours. Nat. Rev. Mater. 2016, 1 (5), 16014.

(9) Mullen, D. G.; Banaszak Holl, M. M. Heterogeneous LigandNanoparticle Distributions: A Major Obstacle to Scientific Understanding and Commercial Translation. Acc. Chem. Res. 2011, 44 (11), $1135-1145$.

(10) Karagoz, B.; Esser, L.; Duong, H. T.; Basuki, J. S.; Boyer, C.; Davis, T. P. Polymerization-Induced Self-Assembly (PISA) - control over the morphology of nanoparticles for drug delivery applications. Polym. Chem. 2014, 5 (2), 350-5.

(11) Niikura, K.; Matsunaga, T.; Suzuki, T.; Kobayashi, S.; Yamaguchi, H.; Orba, Y.; Kawaguchi, A.; Hasegawa, H.; Kajino, K.; Ninomiya, T.; Ijiro, K.; Sawa, H. Gold Nanoparticles as a Vaccine Platform: Influence of Size and Shape on Immunological Responses in Vitro and in Vivo. ACS Nano 2013, 7 (5), 3926-3938.

(12) Dreaden, E. C.; Austin, L. A.; Mackey, M. A.; El-Sayed, M. A. Size matters: gold nanoparticles in targeted cancer drug delivery. 2013, 36.
(13) Choi, C. H. J.; Zuckerman, J. E.; Webster, P.; Davis, M. E. Targeting kidney mesangium by nanoparticles of defined size. Proc. Natl. Acad. Sci. U. S. A. 2011, 108 (16), 6656-61.

(14) Moghimi, S. M.; Hunter, A. C.; Andresen, T. L. Factors Controlling Nanoparticle Pharmacokinetics: An Integrated Analysis and Perspective. Annu. Rev. Pharmacol. Toxicol. 2012, 52 (1), 481503.

(15) Kulkarni, S. A.; Feng, S.-S. Effects of Particle Size and Surface Modification on Cellular Uptake and Biodistribution of Polymeric Nanoparticles for Drug Delivery. Pharm. Res. 2013, 30 (10), 25122522.

(16) Jain, R. K.; Stylianopoulos, T. Delivering nanomedicine to solid tumors. Nat. Rev. Clin. Oncol. 2010, 7 (11), 653-664.

(17) Liu, Y.; Hardie, J.; Zhang, X.; Rotello, V. M. Effects of engineered nanoparticles on the innate immune system. Semin. Immunol. 2017, 34, 25-32.

(18) Gao, H.; Yang, Z.; Zhang, S.; Cao, S.; Shen, S.; Pang, Z.; Jiang, $X . ;$ et al. Ligand modified nanoparticles increases cell uptake, alters endocytosis and elevates glioma distribution and internalization. Sci. Rep. 2013, 3 (1), 2534.

(19) Yao, V. J.; D'Angelo, S.; Butler, K. S.; Theron, C.; Smith, T. L.; Marchio, S.; Gelovani, J. G.; Sidman, R. L.; Dobroff, A. S.; Brinker, C. J.; Bradbury, A. R.M.; Arap, W.; Pasqualini, R. Ligand-targeted theranostic nanomedicines against cancer. J. Controlled Release 2016, 240, 267-86.

(20) Malatesta, M. Transmission electron microscopy for nanomedicine: novel applications for long-established techniques. Eur. J. Histochem [Internet]. 2016 Dec 9 [cited 2020 Apr 30]; 60 (4). Available from: http://www.ejh.it/index.php/ejh/article/view/2751.

(21) Boyd, R. D.; Pichaimuthu, S. K.; Cuenat, A. New approach to inter-technique comparisons for nanoparticle size measurements; using atomic force microscopy, nanoparticle tracking analysis and dyn. Colloids Surf., A 2011, 387, 35-42.

(22) Jee, A.-Y.; Lee, M. Surface functionalization and physicochemical characterization of diamond nanoparticles. Current Applied Physics. 2009, 9 (2), e144-e147.

(23) Rabanel, J.-M.; Adibnia, V.; Tehrani, S. F.; Sanche, S.; Hildgen, P.; Banquy, X.; et al. Nanoparticle heterogeneity: an emerging structural parameter influencing particle fate in biological media? Nanoscale 2019, 11 (2), 383-406.

(24) Woythe, L.; Tito, N. B.; Albertazzi, L. A quantitative view on multivalent nanomedicine targeting. Adv. Drug Delivery Rev. 2020.

(25) Delcanale, P.; Miret-Ontiveros, B.; Arista-Romero, M.; Pujals, S.; Albertazzi, L. Nanoscale Mapping Functional Sites on Nanoparticles by Points Accumulation for Imaging in Nanoscale Topography (PAINT). ACS Nano 2018, 12 (8), 7629.

(26) Feiner-Gracia, N.; Beck, M.; Pujals, S.; Tosi, S.; Mandal, T.; Buske, C.; Linden, M.; Albertazzi, L. Super-Resolution Microscopy Unveils Dynamic Heterogeneities in Nanoparticle Protein Corona. Small 2017, 13 (41), 1701631.

(27) Clemments, A. M.; Botella, P.; Landry, C. C. Spatial Mapping of Protein Adsorption on Mesoporous Silica Nanoparticles by Stochastic Optical Reconstruction Microscopy. J. Am. Chem. Soc. 2017, 139 (11), 3978.

(28) Albertazzi, L.; van der Zwaag, D.; Leenders, C. M. A.; Fitzner, R.; van der Hofstad, R. W.; Meijer, E. W. Probing Exchange Pathways in One-Dimensional Aggregates with Super-Resolution Microscopy. Science 2014, 344 (6183), 491.

(29) Chen, X.; Cui, J.; Ping, Y.; Suma, T.; Cavalieri, F.; Besford, Q. A.; et al. Probing cell internalisation mechanics with polymer capsules. Nanoscale 2016, 8 (39), 17096-101.

(30) Post, R. A. J.; van der Zwaag, D.; Bet, G.; Wijnands, S. P. W.; Albertazzi, L.; Meijer, E. W.; et al. A stochastic view on surface inhomogeneity of nanoparticles. Nat. Commun. 2019, 10 (1), 1663.

(31) Horáček, M.; Engels, D. J.; Zijlstra, P. Dynamic single-molecule counting for the quantification and optimization of nanoparticle functionalization protocols. Nanoscale 2020, 12 (6), 4128-36. 
(32) Pujals, S.; Feiner-Gracia, N.; Delcanale, P.; Voets, I.; Albertazzi, L. Super-resolution microscopy as a powerful tool to study complex synthetic materials. Nat. Rev. Chem. 2019, 3 (2), 68-84.

(33) Jungmann, R.; Steinhauer, C.; Scheible, M.; Kuzyk, A.; Tinnefeld, P.; Simmel, F. C. Single-Molecule Kinetics and SuperResolution Microscopy by Fluorescence Imaging of Transient Binding on DNA Origami. Nano Lett. 2010, 10 (11), 4756-4761.

(34) Agasti, S. S.; Wang, Y.; Schueder, F.; Sukumar, A.; Jungmann, R.; Yin, P. DNA-barcoded labeling probes for highly multiplexed Exchange-PAINT imaging. Chem. Sci. 2017, 8 (4), 3080-91.

(35) Jungmann, R.; Avendano, M. S; Dai, M.; Woehrstein, J. B; Agasti, S. S; Feiger, Z.; Rodal, A.; Yin, P.; et al. Quantitative superresolution imaging with qPAINT. Nat. Methods 2016, 13 (5), 439442.

(36) Delcanale, P.; Albertazzi, L. DNA-PAINT super-resolution imaging data of surface exposed active sites on particles. Data in Brief. 2020, 30, 105468.

(37) Xu, M.; Soliman, M. G.; Sun, X.; Pelaz, B.; Feliu, N.; Parak, W. J.; Liu, S. How Entanglement of Different Physicochemical Properties Complicates the Prediction of in Vitro and in Vivo Interactions of Gold Nanoparticles. ACS Nano 2018, 12, 10104.

(38) Jiang, Y.; Huo, S.; Mizuhara, T.; Das, R.; Lee, Y.-W.; Hou, S.; Moyano, D. F.; Duncan, B.; Liang, X.-J.; Rotello, V. M. The Interplay of Size and Surface Functionality on the Cellular Uptake of Sub-10 nm Gold Nanoparticles. ACS Nano 2015, 9 (10), 9986-93.

(39) de Boer, P.; Hoogenboom, J. P.; Giepmans, B. N. G. Correlated light and electron microscopy: ultrastructure lights up! Nat. Methods 2015, 12 (6), 503-513.

(40) van Elsland, D. M.; Pujals, S.; Bakkum, T.; Bos, E.; Oikonomeas-Koppasis, N.; Berlin, I.; Neefjes, J.; Meijer, A. H.; Koster, A. J.; Albertazzi, L.; van Kasteren, S. I. Ultrastructural Imaging of Salmonella -Host Interactions Using Super-resolution Correlative Light-Electron Microscopy of Bioorthogonal Pathogens. ChemBioChem 2018, 19 (16), 1766-1770.

(41) Kim, D.; Deerinck, T. J.; Sigal, Y. M.; Babcock, H. P.; Ellisman, M. H.; Zhuang, X.; Fox, M. A. Correlative Stochastic Optical Reconstruction Microscopy and Electron Microscopy. PLoS ONE 2015, 10 (4), e0124581.

(42) Watanabe, S.; Punge, A.; Hollopeter, G.; Willig, K. I; Hobson, R. J.; Davis, M W.; Hell, S. W; Jorgensen, E. M Protein localization in electron micrographs using fluorescence nanoscopy. Nat. Methods 2011, 8 (1), 80-84.

(43) Betzig, E.; Patterson, G. H.; Sougrat, R.; Lindwasser, O. W.; Olenych, S.; Bonifacino, J. S.; Davidson, M. W.; Lippincott-Schwartz, J.; Hess, H. F. Imaging Intracellular Fluorescent Proteins at Nanometer Resolution. Science 2006, 313 (5793), 1642-1645.

(44) Tuijtel, M. W.; Koster, A. J.; Jakobs, S.; Faas, F. G. A.; Sharp, T. $\mathrm{H}$. Correlative cryo super-resolution light and electron microscopy on mammalian cells using fluorescent proteins. Sci. Rep. 2019, 9 (1), 1369.

(45) Ando, T.; Bhamidimarri, S. P.; Brending, N.; Colin-York, H; Collinson, L.; De Jonge, N.; de Pablo, P J; Debroye, E.; Eggeling, C.; Franck, C.; Fritzsche, M.; Gerritsen, H.; Giepmans, B. N G; Grunewald, K.; Hofkens, J.; Hoogenboom, J. P; Janssen, K. P F; Kaufmann, R.; Klumperman, J.; Kurniawan, N.; Kusch, J.; Liv, N.; Parekh, V.; Peckys, D. B; Rehfeldt, F.; Reutens, D. C; Roeffaers, M. B J; Salditt, T.; Schaap, I. A T; Schwarz, U. S; Verkade, P.; Vogel, M. W; Wagner, R.; Winterhalter, M.; Yuan, H.; Zifarelli, G. The 2018 correlative microscopy techniques roadmap. J. Phys. D: Appl. Phys. 2018, 51 (44), 443001.

(46) Han, S.; Raabe, M.; Hodgson, L.; Mantell, J.; Verkade, P.; Lasser, T.; Landfester, K.; Weil, T.; Lieberwirth, I. High-Contrast Imaging of Nanodiamonds in Cells by Energy Filtered and Correlative Light-Electron Microscopy: Toward a Quantitative Nanoparticle-Cell Analysis. Nano Lett. 2019, 19 (3), 2178-85.

(47) Le Trequesser, Q.; Deves, G.; Saez, G.; Daudin, L.; Barberet, P.; Michelet, C.; Delville, M.-H.; Seznec, H. Single Cell In Situ Detection and Quantification of Metal Oxide Nanoparticles Using
Multimodal Correlative Microscopy. Anal. Chem. 2014, 86 (15), $7311-7319$.

(48) Rezvantalab, S.; Drude, N. I.; Moraveji, M. K.; Guvener, N.; Koons, E. K.; Shi, Y.; Lammers, T.; Kiessling, F. PLGA-Based Nanoparticles in Cancer Treatment. Front. Pharmacol. 2018, 9, 1260.

(49) Danhier, F.; Ansorena, E.; Silva, J. M.; Coco, R.; Le Breton, A.; Préat, V. PLGA-based nanoparticles: An overview of biomedical applications. J. Controlled Release 2012, 161 (2), 505-522.

(50) Makadia, H. K.; Siegel, S. J. Poly Lactic-co-Glycolic Acid (PLGA) as Biodegradable Controlled Drug Delivery Carrier. Polymers 2011, 3 (3), 1377-1397.

(51) Jokerst, J. V.; Lobovkina, T.; Zare, R. N.; Gambhir, S. S. Nanomedicine 2011, 6 (4), 715-28.

(52) Turecek, P. L.; Bossard, M. J.; Schoetens, F.; Ivens, I. A. PEGylation of Biopharmaceuticals: A Review of Chemistry and Nonclinical Safety Information of Approved Drugs. J. Pharm. Sci. 2016, 105 (2), 460-475.

(53) Vllasaliu, D.; Fowler, R.; Stolnik, S. PEGylated nanomedicines: recent progress and remaining concerns. Expert Opin. Drug Delivery 2014, 11 (1), 139-154.

(54) Nobs, L.; Buchegger, F.; Gurny, R.; Allémann, E. Current methods for attaching targeting ligands to liposomes and nanoparticles. J. Pharm. Sci. 2004, 93 (8), 1980-1992.

(55) Yu, M. K.; Park, J.; Jon, S. Targeting Strategies for Multifunctional Nanoparticles in Cancer Imaging and Therapy. Theranostics 2012, 2 (1), 3-44.

(56) Martinez-Jothar, L.; Doulkeridou, S.; Schiffelers, R. M.; Sastre Torano, J.; Oliveira, S.; van Nostrum, C. F.; Hennink, W. E.; et al. Insights into maleimide-thiol conjugation chemistry: Conditions for efficient surface functionalization of nanoparticles for receptor targeting. J. Controlled Release 2018, 282, 101-109.

(57) Delcanale, P.; Porciani, D.; Pujals, S.; Jurkevich, A.; Chetrusca, A.; Tawiah, K. D.; Burke, D. H.; Albertazzi, L.; et al. Aptamers with Tunable Affinity Enable Single-Molecule Tracking and Localization of Membrane Receptors on Living Cancer Cells. Angew. Chem., Int. Ed. 2020, 59 (42), 18546-18555.

(58) Kiuchi, T.; Higuchi, M.; Takamura, A.; Maruoka, M.; Watanabe, N. Multitarget super-resolution microscopy with highdensity labeling by exchangeable probes. Nat. Methods 2015, 12 (8), 743-746.

(59) Barichello, J. M.; Morishita, M.; Takayama, K.; Nagai, T. Encapsulation of Hydrophilic and Lipophilic Drugs in PLGA Nanoparticles by the Nanoprecipitation Method. Drug Dev. Ind. Pharm. 1999, 25 (4), 471-476.

(60) Martin, F.J.; Papahadjopoulos, D. Irreversible Coupling of Immunoglobulin Fragments to Preformed Vesicles. J. Biol. Chem. 1982, 257 (1), 286-288.

(61) Zhang, J.; Pei, Y.; Zhang, H.; Wang, L.; Arrington, L.; Zhang, Y.; Glass, A.; Leone, A. M.; et al. Assessing the Heterogeneity Level in Lipid Nanoparticles for siRNA Delivery: Size-Based Separation, Compositional Heterogeneity, and Impact on Bioperformance. Mol. Pharmaceutics 2013, 10 (1), 397-405.

(62) Dutta, D.; Salifu, M.; Sirianni, R. W.; Stabenfeldt, S. E. Tailoring sub-micron PLGA particle release profiles via centrifugal fractioning: TAILORING SUB-MICRON PLGA PARTICLE. $J$. Biomed. Mater. Res., Part A 2016, 104 (3), 688-696.

(63) Mullen, D. G.; Borgmeier, E. L.; Desai, A. M.; van Dongen, M. A.; Barash, M.; Cheng, X.-m.; Baker, J. R.; Banaszak Holl, M. M.; et al. Isolation and Characterization of Dendrimers with Precise Numbers of Functional Groups. Chem. - Eur. J. 2010, 16 (35), 10675-10678.

(64) Javiya, C.; Jonnalagadda, S. Physicochemical characterization of spray-dried PLGA/PEG microspheres, and preliminary assessment of biological response. Drug Dev. Ind. Pharm. 2016, 42 (9), 1504-1514.

(65) Vega, E.; Egea; Calpena; Espina; García. Role of hydroxypropyl-\&beta;-cyclodextrin on freeze-dried and gamma-irradiated PLGA and PLGA\&ndash;PEG diblock copolymer nanospheres for ophthalmic flurbiprofen delivery. Int. J. Nanomed. 2012, 1357.

(66) Abstiens, K.; Gregoritza, M.; Goepferich, A. M. Ligand Density and Linker Length are Critical Factors for Multivalent Nanoparticle- 
Receptor Interactions. ACS Appl. Mater. Interfaces 2019, 11 (1),

1311-1320.

(67) Hennig, R.; Pollinger, K.; Veser, A.; Breunig, M.; Goepferich, A. Nanoparticle multivalency counterbalances the ligand affinity loss upon PEGylation. J. Controlled Release 2014, 194, 20-27. 